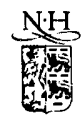

HI SHVIH:R

\title{
Coupled plastic-damaged model
}

\author{
Bibiana Luccioni*, Sergio Oller, Rodolfo Danesi \\ Lahoratorio de Estrucuras. Universidad Nacional de Tucuman - Ar. Roca ISOW. $4(0 \%)$ S.M. de Tucumán. Argentina
}

Received If Junc 1994; revised 21 March 1995

\begin{abstract}
A constitutive model that couples plasticity and damage is presented. The model is thermodynamically consistent and comes from a generalization of classical plasticity theory and isotropic damage theory af Kachanov. Coupling between plasticity and damage is achieved through a simultaneous solution of the plastic and the damage problem. After a description of the model, a numericai algorithm for the integration of the resulting constitutive equations is presented. It is an Euler Backward type of algorithrn that is particularly suitable to solve plain stress nom-linear problems with a 2D finite element program. The consistent stiffnes: matrix is also derived. The paper is completed with some application examples that show that the model presented accurately reproduces the behaviour of elastic-plastic-damaged materials.
\end{abstract}

\section{Introduction}

The greater part of the materials, in particular geomaterials, presents a non-lineiar behaviour accompanied by permanent strains and stiffness degradation when they are subjected to mechanical forces.

In geomaterials, permanent strains are caused by microcracking. Plasticity theory can be used as a mathematical framework to treat these permanent strains if it is considered that they resemble plastic stains $[1,2]$.

Damage or stiffness degradation is related to initiation, growth and interconnection of microcracks and micropores. A great number of approaches have been proposed to simulate this phenomena (see $[3,4]$ ). Among them, continuum damage mechanics has been introduced and widely used to simulate progressive degradation of mechanical properties of materials before the initiation of macro cracking. Kachanov [5] was the first to introduce the concept of effective stress to model creep rupture. Later, continuum damage mechanics was extended to model fatigue. creep, creep-fatigue interaction and ductile-plastic damage. Recently. it has been applied to brittle materials like concrete or rock.

Continuum damage mechanics theories are based on the thermodynamics of irreversible process. Only one scalar variable is needed to model isotropic damage while tensorial variables are needed to model damage anisotropy. For simplicicy. efficiency and adaptation to different practice applications, isotropic formulations are widely used [3].

There is some experimental evidence that damage is linked to plastic strains [3]. Physical mechanisms of interaction between damage and plastic strains are complicated in nature and cannot be modeled by only one phenomenological approach. Little is known about effects of temperature. strain rate, localization or microstructure in these interactions [4]. A great effort was made by some authors to reproduce coupling of damage and plastic processes. Ju [3] gave an energy-based isotropic elastoplastic

\footnotetext{
* Corresponding author.
} 
damage theory that is capable of accommodating non-linear elastic response and general plastic response. The theory can predict degradation in both elastic and plastic responses. This framework was further extended to develop simple energy-based fourth-order anisotropic damage models for brittle materials. Voyiadjis [4] presented a coupled theory of continuum damage mechanics and finite strain plasticity (with small elastic strains). He derived an explicit matrix representation for the damage effect tensor for a general state of deformation and damage. Edlun [6] presented a coupled elastic-plastic damage model for rubber-bond epoxy adhesives. It is a phenomenological model derived from a mathematical framework based on thermodynamic considerations where the micromechanical processes are accounted for by a set of internal variables.

The model presented in this paper is thermodynamically consistent and comes from a generalization of plasticity theory $[1,2]$ and isotropic damage theory. The plastic model is more general than the one in [2] and the treatment of damage is completely different. Coupling of damage and plastic strains is achieved by solving buth problems simultaneously [7]. In this way correct energy dissipation is also assured.

\section{Thermodynamic basis}

The constitutive model proposed is based on the hypothesis of uncoupled elasticity $[8,9]$. According to this hypothesis, the total free energy $\Psi$ can be supposed to be formed by two independent parts: an elastic part $\Psi^{2}$ and a plastic part $\Psi^{\mathrm{r}}$, corresponding to the elastic and plastic processes, respectively

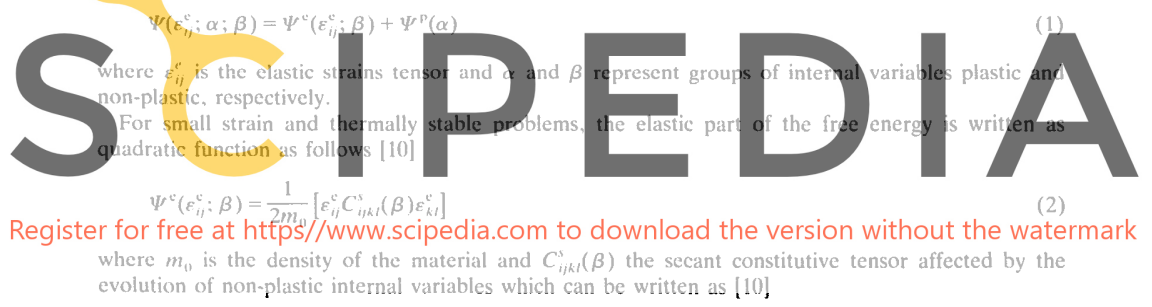

$$
C_{j k k}^{\prime}(\beta)=f(\beta) C_{i j k l}^{\prime \prime}
$$

where $C_{i j k l}^{\prime \prime}$ is the initial secant constitutive tensor of the virgin material and $f(\beta)$ a tensorial transiormation function from an undamaged equivalent space to the real damaged space. The most simple form for the transformation function is the one coinciding with isotropic damage theory of Kachanov [5]

$$
f(\beta)=(1-d)
$$

where $\beta=d$ is the inner damage variable and is such that

$d=0$ for the undamaged virgin material

$d=1$ for the completely damaged material

For this particular choice, the elastic part of the free energy can be written as

$$
\Psi^{c}=(1-d) \Psi^{\prime \prime}=(1-d) \frac{1}{2 m_{0}}\left[\varepsilon_{i j}^{\mathrm{e}} C_{i i k l}^{\prime \prime} \varepsilon_{k l}^{\mathrm{c}}\right]
$$

where $\Psi^{\prime \prime}$ represents the elastic free energy of the undamaged material. 
Inequality of Clasius-Duhem $\{11\}$ can be written in terms of free energy as follows

$$
\Xi=m_{0}(-\psi-\eta \dot{\theta})+\sigma_{t i} \dot{\varepsilon}_{n}-\frac{1}{\theta} q_{r} \frac{\partial \theta}{\partial x_{r}} \geqslant 0
$$

where $\eta$ is the entropy, $\theta$ a measure of temperature, $q$ the heat flow and $x_{i}$ a set of spatial coordinates.

Substituting the form proposed for the free energy (Eq. (1)) in Eq. (2) and splitting the total strain tensor in an elastic and a plastic part, the following inequality is obtained

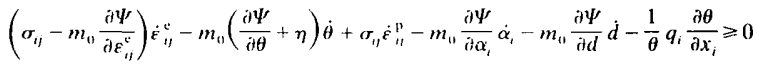

The fulfillment of inequality of Clasius-Planck for a given thermodynamic state is guaranteed if the following equations, known as Coleman [12] relations, are satisfied

$$
\sigma_{i j}=m_{0} \frac{\partial \Psi^{c}}{\partial \varepsilon_{, "}^{c}} \text { and } \eta=-\frac{\partial \Psi}{\partial \theta}
$$

On the other hand, for uncoupled thermomechanical problems, the inequalities of Clasius-Planck must be independently satisfied

\section{(a) Mechanical dissipation}

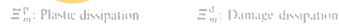

$$
\begin{aligned}
& \Xi_{n}=\sigma_{i j} \dot{\varepsilon}_{i}^{\Gamma}-m_{0} \frac{\partial \psi \Gamma}{\partial \alpha} \dot{\alpha}_{i}-m_{11} \frac{\partial \psi^{c}}{\partial d} \dot{d} \geqslant 0
\end{aligned}
$$

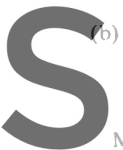

Thermal dissipation

$$
\Xi_{\theta}=\frac{1}{\theta} q, \frac{\partial \theta}{\partial x} \leqslant 0
$$
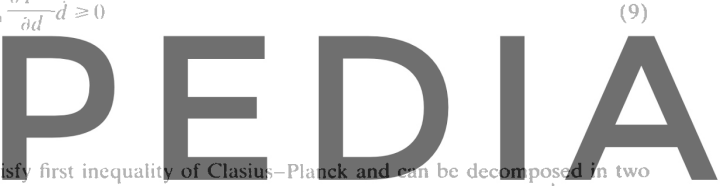

parts: one part due to the plastic process $\Xi_{m}^{\mathrm{r}}$ and the other due to the damage process $\Xi_{m}^{ \pm}$

\section{Register for free at https//www.scipedia.com to download the version without the watermark}

\section{Plastic process}

The plastic process is described by a generalization of classical plasticity theory that takes into account many aspects of geomaterials behaviour.

Elastic threshold is defined by a yield function:

$$
F\left(\sigma_{i,} ; \alpha_{k}\right)=f\left(\sigma_{i j}\right)-K\left(\sigma_{i j} ; \alpha_{k}\right) \leqslant 0
$$

where $f\left(\sigma_{i j}\right)$ is the equivalent tension defined in the damaged space, $K\left(\sigma_{i j} ; \alpha_{k}\right)$ is the equivalent yielding threshold and $\dot{\alpha}_{k}$ a set of internal variables defined as follows

$$
\alpha_{k}=\left\{\kappa^{r} ; \varphi ; K\right\}
$$

where $\kappa^{\Gamma}$ is the plastic damage variable $[1,2]$ and $\varphi$ the angle of internal friction.

The following rules are used for the evolution of plastic strains $\varepsilon_{i j}^{\mathrm{p}}$ and plastic internal variables $\alpha_{k}$

$$
\begin{aligned}
& \dot{\varepsilon}_{\| \prime}^{n}=\dot{\lambda} \frac{\partial G\left(\sigma_{m n} ; \alpha_{h}\right)}{\partial \sigma_{i j}} \\
& \dot{\alpha}_{k}=\dot{\lambda} H_{n}\left(\sigma_{i j} ; \alpha_{m}\right)=\dot{\lambda}\left(h_{k}\right)_{i j} \frac{\partial G\left(\sigma_{m, n} ; \alpha_{l}\right)}{\partial \sigma_{i j}}
\end{aligned}
$$

where $\dot{\lambda}$ is the plastic consistency factor. $G$ the potential function and $\left(h_{k}\right)_{i j}$ a tensor to be defined for each plastic internal variable $[1,2]$. 
The plastic damage variable $\kappa^{p}$ is obtained normalizing energy dissipated by the plastic process to unity [7] and varies from 0 , for the virgin material, to 1 , when the maximum energy if plastically dissipated.

$$
\dot{\kappa}^{\mathrm{p}}=\left[\frac{r}{g_{\mathrm{f}}^{* \mathrm{p}}}+\frac{(1-r)}{g_{\mathrm{c}}^{* \mathrm{p}}}\right] \sigma_{i j} \dot{\varepsilon}_{i j}^{\mathrm{r}}
$$

where

$$
r=\frac{\sum_{i=1}^{3}\left\langle\sigma_{i}\right\rangle}{\sum_{i=1}^{3}\left|\sigma_{i}\right|} \quad\left\langle\sigma_{i}\right\rangle=\frac{1}{2}\left[\sigma_{i}+\left|\sigma_{i}\right|\right],
$$

$\sigma_{i}$ : principal stresses,

$$
g_{i}^{* \mathrm{p}}=\frac{\sum_{i=1}^{3}\left|\sigma_{i}\right| R^{1 \mathrm{pp}}}{f\left(\sigma_{i j}\right)} g_{i}^{\mathrm{p}} \quad g_{\mathrm{c}}^{* \mathrm{p}}=\frac{\sum_{i=1}^{3}\left|\sigma_{i}\right|}{f\left(\sigma_{i j}\right)} g_{\mathrm{c}}^{\mathrm{p}} .
$$

$R^{\prime \prime r}$ is the relation between the yielding threshold in uniaxial compression and that corresponding to uniaxial tension; $g_{f}^{p}$ and $g_{c}^{n}$ are the maximum energy densities dissipated by the plastic process in uniaxial tension and compression processes, respectively. In the case of a thermodynamic process with no damage dissipation, they can be evaluated as follows

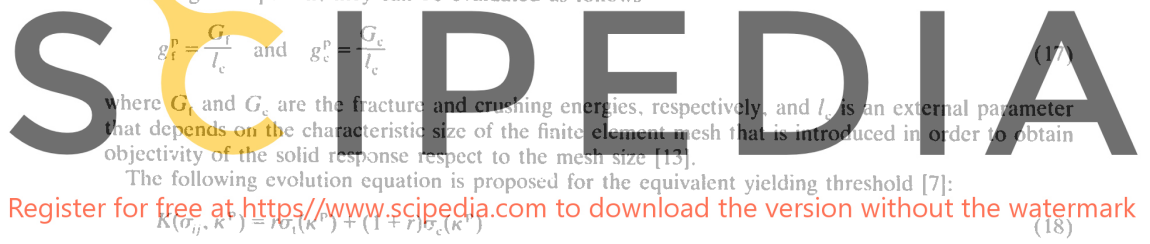

where $\sigma_{1}\left(\kappa^{p}\right)$ and $\sigma_{c}\left(\kappa^{p}\right)$ represent the evolution of the yiclding threshold in uniaxial tension and compression tests, respectively.

Loading/unloading conditions are derived from the Kuhn-Tucker relations formulated for problems with unilateral restrictions:
(a) $\dot{\lambda} \geqslant 0$
(b) $F \leqslant 0$
(c) $\dot{\lambda} F=0$

\section{Damage process}

The damage threshold is described by a damage function in the following way [7]

$$
G^{\mathrm{D}}=\bar{\sigma}\left(\sigma_{i j}\right)-f_{c}\left(\sigma_{i j}, \kappa^{\mathrm{d}}\right) \leqslant 0
$$

where $\bar{\sigma}\left(\sigma_{i j}\right)$ is the equivalent tension defined in the damaged space, $f_{c}\left(\sigma_{i j}, \kappa^{\mathrm{d}}\right)$ is the equivalent damage threshold and $\kappa^{d}$ is the degradation variable.

The equivalent tension $\bar{\sigma}\left(\sigma_{i j}\right)$ can be evaluated using known yiclding functions (Tresca, Von-Mises, Mohr-Coulomb or Drucker-Prager) or any function specially developed for damage. 
The degradation variable varies from 0 , for the virgin material, to 1 , for the completely damaged material and is obtained normalizing energy dissipated by damage to unity [7].

$$
\dot{\kappa}^{\prime \prime}=\left[\frac{r}{g_{i}^{* d}}+\frac{(1-r)}{g_{*}^{* d}}\right] m_{10} \Psi^{\prime \prime} d
$$

where

$$
g_{\mathrm{t}}^{* \mathrm{~d}}=\frac{\sum_{i-1}^{3}\left|\sigma_{i}\right| R^{\mathrm{ik}}}{\bar{\sigma}} g_{1}^{\mathrm{d}} \quad g_{\mathrm{c}}^{* \mathrm{~d}}=\frac{\sum_{i}^{3}\left|\sigma_{i}\right|}{\bar{\sigma}} g_{\mathrm{c}}^{\mathrm{d}},
$$

$R^{\text {ild }}$ is the relation between the damage threshold in uniaxial compression and that corresponding to uniaxial tension and $g_{\mathrm{r}}^{\mathrm{d}}$ and $g_{\mathrm{c}}^{\mathrm{d}}$ are maximum energy densities dissipated by damage in uniaxial tension and compression processes, respectively.

The following evolution equation is proposed for the equivalent damage threshold:

$$
f_{c}\left(\sigma_{i,}, \kappa^{d}\right)=r \sigma_{i}\left(k^{d i}\right)+(1-r) \sigma_{i}\left(\kappa^{\prime \prime}\right)
$$

where $\sigma_{1}\left(\kappa^{\mathrm{d}}\right)$ and $\sigma_{\mathrm{e}}\left(\kappa^{\mathrm{d}}\right)$ represent the evolution of the damage threshold in uniaxial tension and compression tests, respectively.

The loading/unloading conditions are derived from the Kuhn-Tucker relations and are analogous to the ones corresponding to the plastic process:
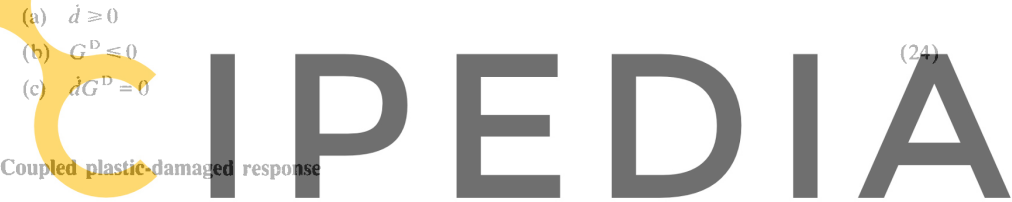

Evolution of permanent strains and damage is obtained from the simultaneous solution of the

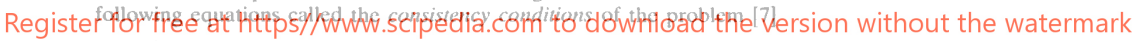

$$
\begin{aligned}
& \dot{F}=0 \\
& \dot{G}^{\mathrm{D}}=0
\end{aligned}
$$

Eqs. (25) are two linear equations in $\dot{\lambda}$ and $\dot{d}$ that can be easily solved.

The secant constitutive law can be written as follows

$$
\begin{aligned}
& \sigma_{i j}=m_{i} \frac{\partial \Psi^{\mathrm{e}}}{\partial \varepsilon_{i j}^{\mathrm{e}}}=C_{i j k l}^{\mathrm{s}} \varepsilon_{k l}^{\mathrm{c}}=(1-d) C_{i j k l}^{0}\left(\varepsilon_{k l}-\varepsilon_{k l}^{\mathrm{p}}\right) \\
& \sigma_{i j}=(1-d) \sigma_{i j}^{\mathrm{u}}
\end{aligned}
$$

where $\sigma_{i j}^{\prime \prime}$ is the stress tensor of a fictitious undamaged solid. Eq. (27) can be interpreted as a transformation between the real damaged space an a fictitious undamaged space.

Tangent constitutive law can be obtained from Eqs. (25) and (26) and results

$$
\dot{\sigma}_{i j}=C_{i j k l}^{t} \dot{\varepsilon}_{k l}
$$

where

$$
C_{i j k l}^{\prime}=C_{i j k l}^{e}-\frac{C_{i j r s}^{s} \frac{\partial G}{\partial \sigma_{r s}} \frac{\partial F}{\partial \sigma_{m n}} C_{m n k l}^{c}}{-\frac{\partial F}{\partial \alpha_{p}}\left(h_{p}\right)_{r t} \frac{\partial G}{\partial \sigma_{i t}}+\frac{\partial F}{\partial \sigma_{m n}} C_{m n r s}^{s} \frac{\partial G}{\partial \sigma_{r s}}}
$$


and

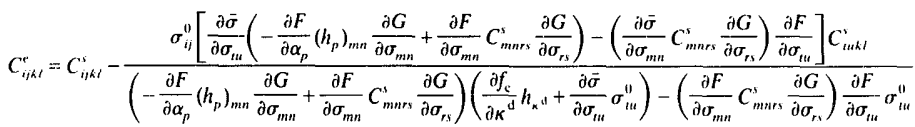

\section{Algorithm for the numerical implementation of the plastic-damaged model}

Capacity of a constitutive model to predict solid response not only depends on the model itself but also on the numerical integration of the model. The precision with which constitutive equations are integrated has a direct impact on the accuracy of the results $[14,15]$.

For the proposed model, yiclding and damage equations must be integrated simultancenusly. An Euler-Backward algorithm $[7,16,17]$ is presented for this purpose. Solution of plane stress problems with this type of methods is straightforward. Between two equilibrium configurations $n$ and $n-1$ the variables of the problem are updated as follows

$$
\left(\varepsilon_{i j}^{\mathrm{r}}\right)_{n}=\left(\varepsilon_{i j}^{\mathrm{P}}\right)_{n-1}+\Delta \lambda\left(\frac{\partial G}{\partial \sigma_{i j}}\right)_{n}
$$

$$
\left(\alpha_{i}\right)_{n}=\left(\alpha_{i}\right)_{n-1}+\Delta \lambda\left(H_{i}\right)_{n}
$$
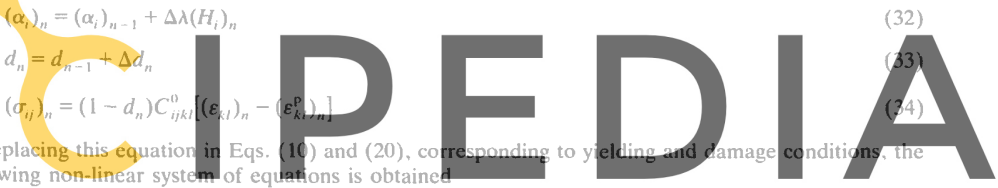

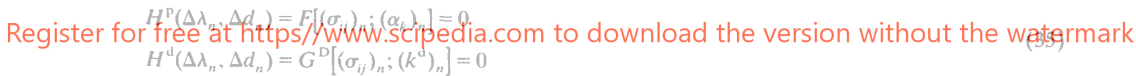

This system can be solved, for example, by the Newton-Raphson method

$$
\left\{\begin{array}{l}
\Delta \lambda_{n} \\
\Delta d_{n}
\end{array}\right\}^{k}=\left\{\begin{array}{ll}
\Delta \lambda_{n} \\
\Delta d_{n}
\end{array}\right\}^{k-1}-\left[\begin{array}{ll}
\left(\frac{\partial H^{\mathrm{P}}}{\partial \Delta \lambda}\right)_{n} & \left(\frac{\partial H^{\mathrm{P}}}{\partial \Delta d}\right)_{n} \\
\left(\frac{\partial H^{\mathrm{d}}}{\partial \Delta \lambda}\right)_{n} & \left(\frac{\partial H^{\mathrm{d}}}{\partial \Delta \lambda}\right)_{n}
\end{array}\right]^{k-1-1}\left\{\begin{array}{l}
H^{\mathrm{P}}\left(\Delta \lambda_{n}, \Delta d_{n}\right) \\
H^{\mathrm{d}}\left(\Delta \lambda_{n}, \Delta d_{n}\right)
\end{array}\right\}^{k-1}
$$

The algorithm is summarized in Table 1.

Some recent publications [16-19] pointed out the advantage of using a consistent stiffness matrix when solving elastoplastic problems. It has been proved that the quadratic rate of convergence of an incremental solution based on a Newton-Raphson procedure can only be ensured if the tangent modulus is derived in a way consistent with the constitutive integration algorithm.

The consistent tangent modulus corresponding to the plastic-damaged model and the algorithm proposed is developed below.

Differentiating Eq. (34) and taking into account Eqs. (31)-(33), it results

$$
\begin{aligned}
\left(\mathrm{d} \sigma_{i j}\right)_{n}= & -d\left(d_{n}\right) C_{i j k l}^{0}\left[\left(\varepsilon_{k l}\right)_{n}-\left(\varepsilon_{k l}^{\mathrm{p}}\right)_{n-1}-\Delta \lambda_{n}\left(\frac{\partial G}{\partial \sigma_{k l}}\right)_{n}\right] \\
& +\left(C_{i j k l}^{s}\right)_{n}\left(\mathrm{~d} \varepsilon_{i j}\right)_{n}-\mathrm{d} \lambda_{n}\left(C_{i j k l}^{s}\right)_{n}\left(\frac{\partial G}{\partial \sigma_{k l}}\right)_{n}-\Delta \lambda_{n}\left(C_{i j k l}^{s}\right)_{n}\left(\frac{\partial^{2} G}{\partial \sigma_{k l} \partial \sigma_{p q}}\right)_{n}\left(\mathrm{~d} \sigma_{p q}\right)_{n}
\end{aligned}
$$


Table 1

Algorithm for the numerical integration of eonstitutive equations

(1) Initialization: $k=0 ; \Delta \lambda_{n}^{\prime \prime}=0: \Delta d_{n}^{\prime \prime}=0$

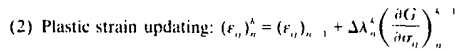

(3) Damage variable updating: $d_{n}^{h}=d_{n},+\Delta d_{n}^{h}$

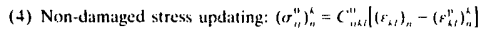

(5) Damaged stress updating: $\left(\sigma_{t,}\right)_{n}^{k}=\left(1-d_{n}^{k}\right)\left(\sigma_{t^{\prime}}^{\prime \prime}\right)_{n}^{k}$

(6) Updating of the other internal variables: $\left(\alpha x_{1}\right)_{n}^{k}=\left(\alpha_{1}\right)_{n}+\Delta \lambda_{n}^{\alpha}\left(H_{1}\right)_{n}^{h}$

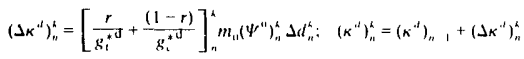

(7) Yielding and damage conditions: If $\left(H^{n}\right)_{n}^{h}=0$ and $\left(H^{4}\right)_{n}^{h} \leq 0$ goto(13)

(8) If $\left(H^{n}\right)_{n}^{\wedge} \geqslant 0$ goto $(10)$

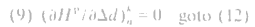

(10) If $\left(H^{\prime \prime}\right)_{n}^{k} \geqslant 0$ goto $(12)$

(11) $\left(i H^{4} / i \Delta \lambda\right)_{n}^{n}=0$

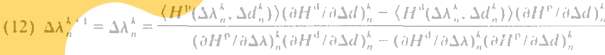

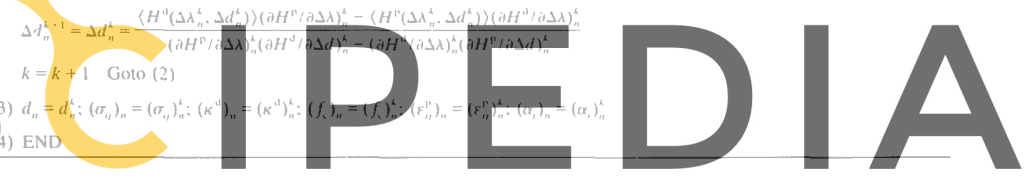

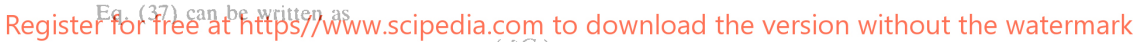

$$
\left(\mathrm{d} \sigma_{i j}\right)_{n}=\left(C_{i j k l}^{i^{*} *}\right)_{n}\left(\mathrm{~d} \varepsilon_{k l}\right)_{n}-\left(C_{i, k l}^{s *}\right)_{n} \mathrm{~d} \lambda_{n}\left(\frac{\partial G}{\partial \sigma_{k l}}\right)_{n}
$$

where

$$
\begin{aligned}
& \left(C_{i j k l}^{r^{*} *}\right)=\left[\delta_{i p} \delta_{i q}+\Delta \lambda_{n}\left(C_{i j r s}^{s}\right)_{n}\left(\frac{\partial^{2} G}{\partial \sigma_{r s} \partial \sigma_{p q}}\right)_{n}\right]^{-1} C_{p q k l}^{c^{\prime}} \\
& \left(C_{i j k l}^{s *}\right)=\left[\delta_{i p} \delta_{j p}+\Delta \lambda_{n}\left(C_{i j r s}^{s}\right)_{n}\left(\frac{\partial^{2} G}{\partial \sigma_{r s} \partial \sigma_{p q}}\right)_{n}\right]^{-1} C_{p i k l}^{s}
\end{aligned}
$$

The consistent tangent modulus results

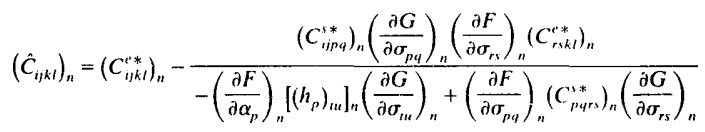

\section{Application examples}

\subsection{Concrete under biaxial compression}

In this example the behavior of concrete under biaxial compression [20] is studied. Concrete was modeled as an elasto-plastic-damaged material. Mechanical properties of concrete are summarized in Table 2. finite element mesh and loading conditions are represented in Fig. 1. The problem was solved 

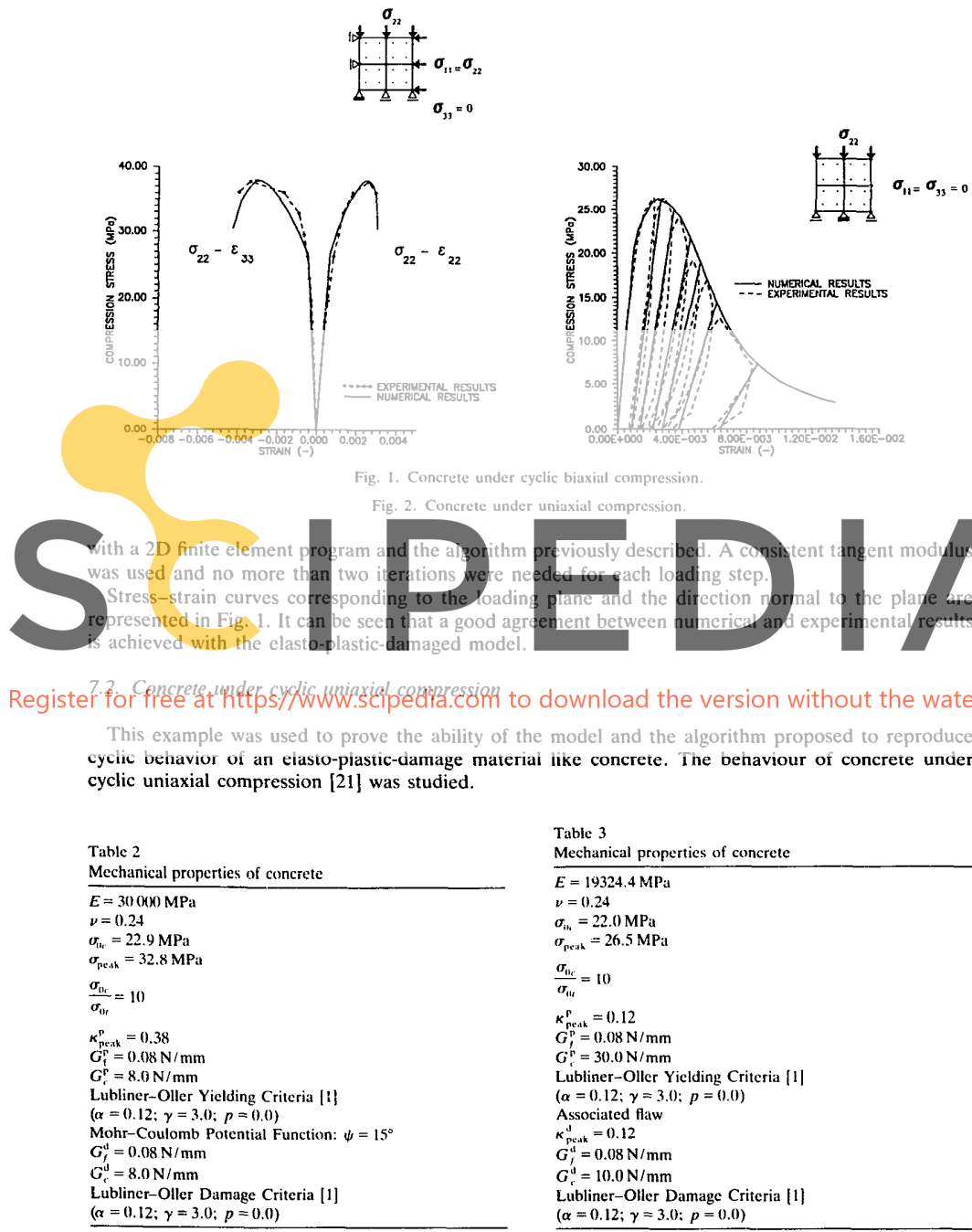

Fig. 2. Concrete under uniaxial compression.

ith a 2D finite element program and the alzon ithm previously described. A consistent tangent modulus as used and no more than two iterations were needed for cach loading step. Stress-strain curves corresponding to the loading plane and the direction no achieved with the elasto-plastic-damaged model.

hent between umerical an ormal to the plane are i experimental results

Mechanical properties of conciete

\begin{tabular}{|c|}
\hline 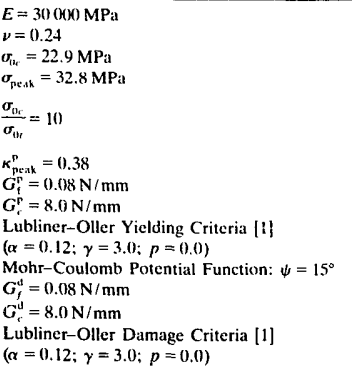 \\
\hline
\end{tabular}

Table 3

Mechanical properties of concrete

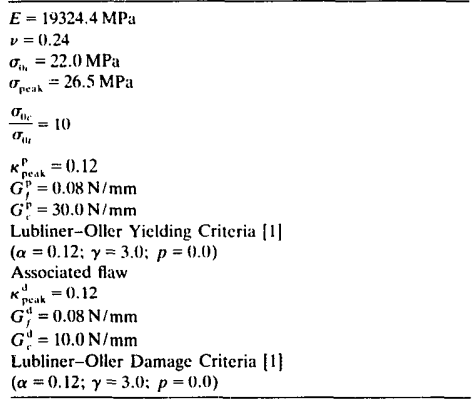


The finite element mesh and loading conditions are represented in Fig. 2. Concrete was modeled as an elasto-plastic-damaged material with the mechanical properties summarized in Table 3.

Experimental and numerical stress-strain curves are represented in Fig. 2. It can be seen that the model accurately reproduces the behavior of concrete. Unloading branches have almost the same average modulus. Obviously. the model is not able to reproduce hysteresis loops appearing in experimental results because linear elastic behaviour was assumed for unloading cases.

\section{Concluding remarks}

The constitutive model presented, simultaneously solves the problem of evolution of permanent strains and stiffness degradation. Yielding and damage consistent conditions are both satisfied in each loading step. In this way, using hardening variables related to energy dissipation in each problem, correct energy dissipation of the whole problem is achieved.

The model is simple and presents a total analogy with other elasto-plastic models used to reproduce the behaviour of frictional materials.

The algorithm presented for the numerical implementation of the model proposed is a generalization of Euler-Backward algorithms commonly used in plasticity to the case of plasticity coupled with damage. It is particularly suitable for plane stress problems. The consistent tangent modulus developed preserves quadratic tate of convergence of Newton-Raphson method.

\section{References}

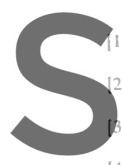
S. Oller, Un Modelo de Daño Continuo
Escola Tecnica Superior DEnginyers de
. Lubliner. J. Oliver, S. Olker and E.
299-326.
W.W. Ju, On energy-based coupled el sisep
Solids Struct. 25(7) (1989) $803-833$.
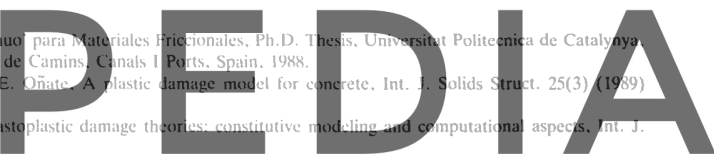

[4] G.Z. Voyiadjis and P.I. Kattan. A plas

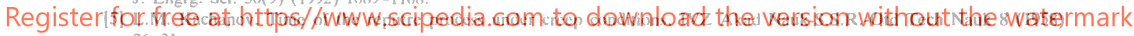
26-31

[6] U. Edlund and A. Klarbring. A coupled elastic-plastic damage model for rubher-modified epoxy adhesives, Int. J. Solids Struct. 30) 19) (1993) 2693-2708.

[7] B.M. Luccioni. Formulación de un modelo constitutive para materiales ortótropos. Ph.D. Thesis. Universidad Nacional de Tucumán. Argentina, 1993.

[8] A. Green and P. Naghdi. A general theory for an elastic-plastic continuum. Arch. Rational Mech. Anal. 18 (1964) 19-281.

[9] J. Lubliner, Plasticity Theory (MacMillan, New York, 1990).

[10] S. Oller, J. Oliver, M. Cervera and J. Oñate, Simulación de procesos de localización en mecánica de sólidos, mediante un modelo plástico. Memorias del l Congreso de Métodos Numéricos en Ingenieria, SEMNI 1990 (1990) 423-431.

[11] L.E. Malvern, Introduction to the Mechanics of Continuous Medium (Prentice Hall, Englewood Cliffs, NJ, 1969).

[12] J. Lubliner. On the thermodynamic foundations of non-linear mechanics, Int. J. Non Linear Mech. 7 (1972) $237-254$.

[13] J. Oliver. A consistent characteristic length for smeared cracking mode's, Int. J. Numer. Methods Engrg. 28 (1989) $461-474$.

[14] M. Ortiz and E.P. Popov. Accuracy and stability of integration algorithms for elastoplastic constitutive relations. Int. J. Numer. Methods Engrg. 21 (1985) 1561-1576.

[15] M. Ortiz and J.C. Simo. An analysis of a new class of integration algorithms for elasto-plastic constitutive relations, Int. J. Nunner. Methods Engrg. 83 (1986) 353-366.

[16] J.C. Simo and T.J.R. Hughes, Elastoplasticity and viscoplasticity, Computational Aspects (Springer-Verlag. Berlin) 97-137. in press

[17] M.A. Crisfield. Non-Linear Finite Element Analysis of Solids and Structures (John Wiley \& Sons Lts., England, 1991).

[18] J.C. Simo and R.L. Taylor, Consistent tangent operators for rate independent clastoplasticity. Comput. Methods Appl. Mech. Engrg. 48 (1985) 101- 118.

[19] G.P. Mitchell and D.R.J. Owen, Numerical solutions for elasto-plastic problems. Engrg. Comput. 5 (1988) $274-284$.

[20] H. Kupfer, H. Hidsford and H. Rusch. Behaviour of concrete under biaxial stresses, J. ACl 66(8) (1969) 656-666.

[21] B.P. Sinha, K.H. Gerstle and L.G. Tulin. Stress-strain relations for concrete under cyclic loading, J. ACl 62(2) (1964) 195-210. 